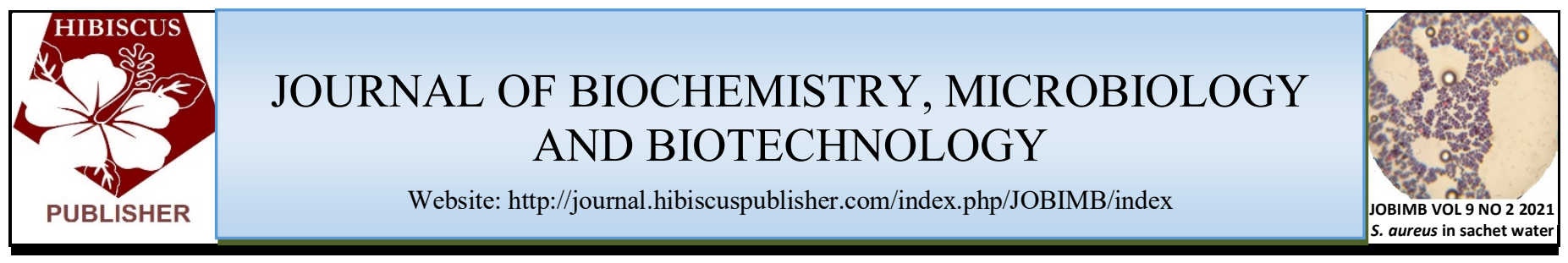

\title{
Kinetic Analysis of the Adsorption of Malachite Green onto Graphene Oxide Sheets Integrated with Gold Nanoparticles
}

\author{
Ibrahim Alhaji Sabo ${ }^{1}$ Salihu Yahuza ${ }^{2}$, Bilal Ibrahim Dan-Iya ${ }^{3 *}$ and Abdussamad Abubakar ${ }^{4}$ \\ ${ }^{1}$ Department of Microbiology, Faculty of Pure and Applied Sciences, Federal University Wukari, P.M.B. 1020 Wukari, \\ Taraba State Nigeria. \\ ${ }^{2}$ Department of Microbiology and Biotechnology, Faculty of Science, Federal University Dutse, P.M.B., 7156, Dutse, \\ Jigawa State, Nigeria. \\ ${ }^{3}$ College of Health Sciences and Technology Kano, Kano, Nigeria. \\ ${ }^{4}$ Department of Microbiology, Faculty of Science, Bauchi State University, Gadau, PMB 65, Nigeria.
}

\author{
*Corresponding author: \\ Bilal Ibrahim Dan-Iya, \\ College of Health Sciences and Technology Kano, \\ Kano, \\ Nigeria. \\ Email: bidaniya@gmail.com
}

\section{HISTORY}

Received: $24^{\text {th }}$ Sep 2021

Received in revised form: $15^{\text {th }}$ Nov 2021 Accepted: $24^{\text {th }}$ Dec 2021

\section{KEYWORDS}

Adsorption, Kinetics

Cibacron blue

Bean peel

Nonlinear regression

Pseudo-2 ${ }^{\text {nd }}$ order

\begin{abstract}
Malachite green is extensively used in the textile dye industry and in agriculture as fish pests' pesticide. Biosorption is a type of sorption technique that uses a biological sorbent. As of now, biosorption is viewed as a simple and cost-effective process that might be used as an alternative to traditional pollution treatment methods. Bioremediation is one of the branches of bioremediation that is used to minimise pollution in the context of incorrect textile waste disposal. The sorption isotherm of Malachite Green onto graphene oxide were analyzed using three models - pseudo- $1^{\text {st }}$, pseudo- $2^{\text {nd }}$ and Elovich, and fitted using non-linear regression. The Elovich model was the poorest in fitting the curve based on visual observation and the best was pseudo$2^{\text {nd }}$ order based on statistical analysis such as root-mean-square error (RMSE), adjusted coefficient of determination $\left(\operatorname{adj} R^{2}\right)$, bias factor $(\mathrm{BF})$, accuracy factor $(\mathrm{AF})$, corrected AICc (Akaike Information Criterion), Bayesian Information Criterion (BIC) and Hannan-Quinn information criterion (HQC). Nonlinear regression analysis using the pseudo- $2^{\text {nd }}$ order model gave values of equilibrium sorption capacity $q_{e}$ of $6.164 \mathrm{mg} / \mathrm{g}(95 \%$ confidence interval from 5.918 to 6.410$)$ and a value of the pseudo- $2^{\text {nd }}$-order rate constant, $k_{2}$ of 0.034 (95\% confidence interval from 0.024 to 0.045 ). Further analysis is needed to provide proof for the chemisorption mechanism usually tied to this kinetic.
\end{abstract}

\section{INTRODUCTION}

Dry and wet techniques are used in the textile business to produce fibres. There are two main types of processing: dry and wet. The dry process encompasses sourcing, bleaching, dyeing, and finishing. Effluents from such phases are heavily polluted since they need a big volume of clean water. The dying process is critical to the efficient interchange of textiles. Product quality attributes such as high optical fixation are sought for by consumers both throughout the purchase process and after longterm use of a certain product. Choosing fibre colourants is a complex process since they need to be precise, color-consistent, fade-resistant, and cost-effective [1].

Dyes and pigments are selected for use in supplies based on their chemical composition and grouping, as well as their commercial affordability, adhesion to the required material, and economical factors. The process of advanced dyeing technology includes a number of steps. Dyeing and pigmenting are two forms of sophisticated dyeing technologies [2,3]. The dyeing methods have changed from time to time, but they haven't progressed much. As a whole, the process is divided into preparation, dyeing, and finishing.

Malachite Green (MG), also known as triphenylmethane dye, is a multipurpose substance that is mostly utilised in the textile industry and is also employed in aquaculture in the form of fungicides and ectoparasiticides. Despite the fact that the effects of MG on aquatic invertebrates and algae are still being studied, MG in wastewater from either industry or aquaculture has been widely reported to be toxic to many species of fish, with lethal effects reported at concentrations as low as $1 \mathrm{mg} / \mathrm{L}$, with the dye and its derivatives being accumulated in aquaculture products such as fish, prawn, and crab. It also contains carcinogenic and genotoxic qualities, which make it a possible health hazard for people [1-4]. As a result, this dye has been prohibited in Europe, the United States, and a number of other nations. However, MG is still in use in various areas of the globe due to the fact that it is very effective and readily accessible at a reasonable price. It is also used in the United States as a cure for 
tropical fish ailments, and since it is easily accessed by the general population, there is worry regarding its unlawful usage. In Asian nations, it has been claimed that $\mathrm{MG}$ is employed in fish aquaculture for the elimination of external parasites and fungal infections. The removal of manganese from aquaculture effluent, on the other hand, has received little or no attention in comparison to the removal of other contaminants. As a result, it is possible that MG contamination in aquaculture effluent may occur, with potentially severe repercussions for the surrounding ecosystem [5-8].

A variety of biotreatment processes, both environmentally friendly and conventional, rely on biosorption techniques. Plants, marine algae, and agricultural waste are only some of the biological materials that may be used to remove organic or inorganic matter from solutions. They can also be used to harvest organic matter from the environment. In a previous study, the sorption of Malachite Green onto graphene oxide was studied using linearized kinetic models [9] which can potentially disrupt the error structure of the data and hindered efficient inference and comparison with current biosorption data that have begun to capitalize on the computing power that allow nonlinear regression to be carried out at ease.

Understanding the mechanism of biosorption necessitates accurate assignment of biosorption kinetics and isotherms. This is particularly true when it comes to understanding biosorption's mechanism. By applying linearization to an obviously nonlinear curve in order to smooth it out, the error structure of the data gets thrown off. Because of this, assessing the uncertainty of the parameters of the kinetics, which is often presented in the form of a 95 percent confidence interval range, becomes significantly more difficult [10]. In addition, the linearization technique results in the introduction of error into the independent variable as a result of the linearization procedure. Furthermore, variations in the weights allocated to each data point may occur, which, according on the data set, often leads in disparities in the fit parameter values between both the linear and nonlinear versions of the kinetics model, respectively [11]. Thus, the aim of this study is to remodel the data using nonlinear regression.

\section{METHODS}

\section{Data acquisition and fitting}

Data from Figure 4 from a published work [9] were digitized using the software Webplotdigitizer 2.5 [12]. The data were then nonlinearly regressed using the curve-fitting software CurveExpert Professional software (Version 1.6). Digitization using this software has been acknowledged for its reliability $[13,14]$. The data were then nonlinearly regressed using the curve-fitting software CurveExpert Professional software (Version 1.6) using several models (Table 1).

Table 1. Kinetic models utilized in this study.

\begin{tabular}{lcl}
\hline Model & Equation & Reference \\
\hline Pseudo- $1^{\text {st }}$ order & $q_{t}=q_{e}\left(1-e^{-K_{1} t}\right)$ & {$[15]$} \\
Pseudo-2 ${ }^{\text {nd }}$ order & $q_{t}=\frac{K_{2} q_{e}{ }^{2} t}{\left(1+K_{2} q_{e} t\right)}$ & {$[16]$} \\
Elovich & $q_{t}=\frac{1}{\beta \ln \alpha \beta}+\frac{1}{\beta \ln t}$ & {$[17]$}
\end{tabular}

\section{Statistical analysis}

Error function analysis is very important to find the best model. Functions such as teh corrected AICc (Akaike Information Criterion), Bayesian Information Criterion (BIC), Hannan and Quinn's Criterion (HQ), Root-Mean-Square Error (RMSE), bias factor (BF), accuracy factor (AF) and adjusted coefficient of determination $\left(R^{2}\right)$ were utilized in this work.

The RMSE was calculated according to Eq. (1), [10], and smaller number of parameters is expected to give a smaller RMSE values. $n$ is the number of experimental data, $O b_{i}$ and $P d_{i}$ are the experimental and predicted data while $p$ is the number of parameters.

$R M S E=\sqrt{\frac{\sum_{i=1}^{n}\left(P d_{i}-O b_{i}\right)^{2}}{n-p}}$

(Eqn. 1)

As $R^{2}$ or the coefficient of determination ignores the number of parameters in a model, the adjusted $R^{2}$ is utilized to overcome this issue. In the equation (Eqns. 2 and 3), the total variance of the y-variable is denoted by ${ }^{2}$ while RMS is the Residual Mean Square.

Adjusted $\left(R^{2}\right)=1-\frac{R M S}{s_{Y}^{2}}$

$\operatorname{Adjusted}\left(R^{2}\right)=1-\frac{\left(1-R^{2}\right)(n-1)}{(n-p-1)}$

The AICc is calculated as follows (Eqn. 4), where $p$ signifies the quantity of parameters and $n$ signify the quantity of data points. To handle data having a high number of parameters or a smaller number of values corrected Akaike information criterion (AICc) is utilized [18].A model with a smaller value of $\mathrm{AICc}$ is deemed likely more correct [18]. The Akaike Information Criterion (AIC) is based on the information theory. It balances between the goodness of fit of a particular model and the complexity of a model [19].

$A I C c=2 p+n \ln \left(\frac{R S S}{n}\right)+2(p+1)+\frac{2(p+1)(p+2)}{n-p-2}$

(Eqn. 4)

Aside from AICc, Bayesian Information Criterion (BIC) (Eqn. 5) is another statistical method that is based on information theory. This error function penalizes the number of parameters more strongly than AIC [20].

$B I C=n \cdot \ln \frac{R S S}{n}+k \cdot \ln (n)$

(Eqn. 5) 
A further error function method based on the information theory is the Hannan-Quinn information criterion (HQC) (Eqn. 6). The HQC is strongly consistent unlike AIC due to the $\ln \ln n$ term in the equation [18];

$$
H Q C=n \times \ln \frac{R S S}{n}+2 \times k \times \ln (\ln n)
$$

Further error function analysis that originates from the work of Ross [21] are the Accuracy Factor (AF) and Bias Factor (BF). These error functions test the statistical evaluation of models for the goodness-of-fit but do not penalize for number of parameter (Eqns. 7 and 8).

Bias factor $=10^{\left(\sum_{i=1}^{n} \log \frac{\left(P d_{i} / O b_{i}\right)}{n}\right)}$

Accuracy factor $=10^{\left(\sum_{i=1}^{n} \log \frac{\left|\left(P d_{i} / O b_{i}\right)\right|}{n}\right)}$

(Eqn. 8)

Another error function analysis is the evidence ratio regarding the difference between the two lowest AICc values (Eqn. 9), where if it is the same, then each model will have an equal chance of being true. If the difference in AICc scores is 6.0, model A has a $95 \%$ chance of being correct, making it $20(95 / 5)$ times more likely than model B to be correct [10].

$P_{A}=\frac{e^{0.5 \Delta}}{1+e^{0.5 \Delta}}$

\section{RESULTS AND DISCUSSION}

Since 3000 B.C., people have employed dyes for a variety of purposes in their daily lives. Among the countries with a thriving dying industry are Egypt, Romania, and India. These colour compounds are regarded as significant pollutants and may be called "discernible contaminants." An estimated 10103 metric tonnes of commercial dyes and pigments are produced each year around the world, with the United States contributing about 70 104 metric tonnes of this total (Slama et al., 2021; Garg, 2004).

Local dyes were used to colour fabrics in ancient times, but the colour palette was limited and the variety of colours was limited. In addition, repeated washing and exposure to sunshine degrade the material's colour fastness over time (Kant, 2011). As a result, mordants were used instead, which necessitated a lengthy dying process. Fabrics could be produced in a wide range of colours and hues thanks to W. H. Perkins's first artificial dyes, which were developed in the $1850 \mathrm{~s}$. As a result, the "dye application" industry is now worth billions of dollars. Synthetic dyes, on the other hand, are losing favour due to the harmful and unpleasant effects they have on all life forms around the world. There is the potential for dyes to be poisonous and to have a significant detrimental impact on effluent quality. The natural dye painting procedure necessitated a significant amount of water. There will be almost as much dye as there is coloured fibre, if not more, in the final product. A garment is dyed with around $80 \%$ of the colourant remaining on the fabric, while the remaining $20 \%$ is flushed away as effluent (Essawy et al., 2008). About $22 \%$ of the country's industrial wastewater comes from the textile industry, according to industry estimates (Tan et al., 2008).
The absorption kinetics data were analyzed using three models pseudo- $1^{\text {st }}$, pseudo- $2^{\text {nd }}$ and Elovich, and fitted using non-linear regression. The Elovich model was the poorest in fitting the curve based on visual observation followed by the Pseudo-1st order (Figs. 1-3). Statistical analysis based on root-mean-square error (RMSE), adjusted coefficient of determination $\left(\operatorname{adj} R^{2}\right)$, bias factor (BF), accuracy factor (AF), corrected AICc (Akaike Information Criterion), Bayesian Information Criterion (BIC) and Hannan-Quinn information criterion (HQC) that showed that the pseudo-2nd-order model was the best (Table 2) which was the same finding from the original published work.

The calculated evidence ratio was 16.7 with an AICc probability value of 0.94 indicating that the best model was at least 16.7 times better than the nearest best model, which was pseudo- $1^{\text {st }}$. Further analysis is needed to provide proof for the mechanism usually tied to this kinetic. Nonlinear regression analysis using the pseudo- $2^{\text {nd }}$ order model gave values of equilibrium sorption capacity $q_{e}$ of $6.164 \mathrm{mg} / \mathrm{g}(95 \%$ confidence interval from 5.918 to 6.410$)$ and a value of the pseudo- $2^{\text {nd }}$-order rate constant, $k_{2}$ of 0.034 (95\% confidence interval from 0.024 to 0.045). In comparison, the published work reported an equilibrium sorption capacity $q_{e}$ of $6.57 \mathrm{mg} / \mathrm{g}$ and a value of the pseudo- $2^{\text {nd }}$-order rate constant, $k_{2}$ of 0.0199 [9]. Further analysis is needed to provide proof for the chemisorption mechanism usually tied to this kinetic.

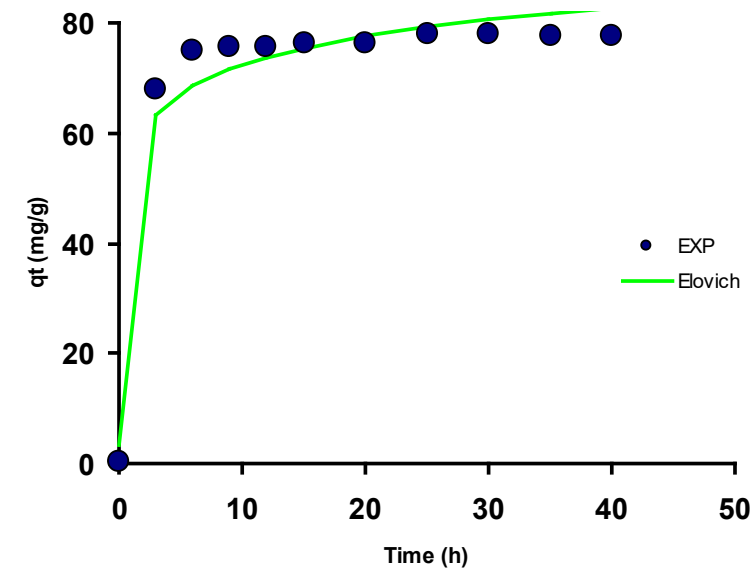

Fig. 1. Kinetics of the sorption of Malachite Green onto graphene oxide as modelled using the Elovich model.

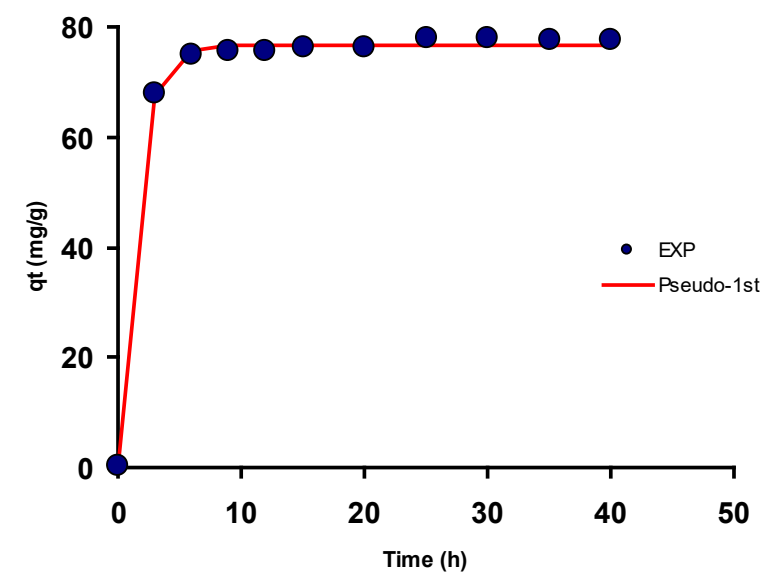

Fig. 2. Kinetics of the sorption of Malachite Green onto graphene oxide as modelled using the pseudo- $1^{\text {st }}$ order model. 


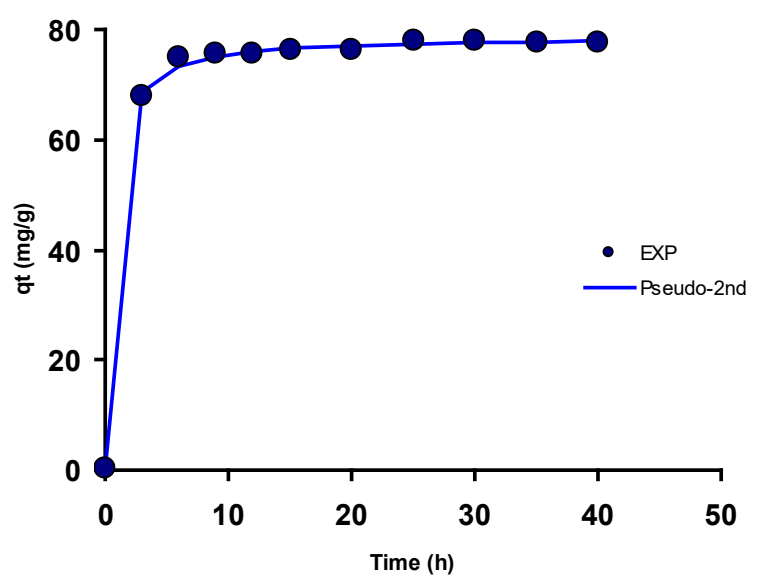

Fig. 3. Kinetics of the sorption of Malachite Green onto graphene oxide as modelled using the pseudo- $2^{\text {nd }}$ order model.

Table 2. Error function analysis of regressed models.

\begin{tabular}{|c|c|c|c|c|c|c|c|}
\hline Model & $\mathrm{p}$ & RMSE & $a d R^{2}$ & $\mathrm{AICc}$ & $\mathrm{BIC}$ & $\mathrm{HQC}$ & $\mathrm{AF}$ \\
\hline Pseudo-1st order & 2 & 0.991 & 0.998 & 11.02 & 2.39 & 1.09 & 1.0101 .000 \\
\hline Pseudo-2nd order & 2 & 0.768 & 0.999 & 5.40 & -3.23 & -4.53 & 1.0071 .000 \\
\hline Elovich & 2 & 3.991 & 0.965 & 41.67 & 33.04 & 31.74 & 1.0370 .976 \\
\hline \multicolumn{8}{|l|}{ Note: } \\
\hline \\
\hline \multicolumn{8}{|c|}{$p \quad$ no of parameters } \\
\hline \multicolumn{8}{|c|}{ Adjusted Coefficient of determination } \\
\hline \multicolumn{8}{|l|}{ Bias factor } \\
\hline \multicolumn{8}{|c|}{ Accuracy factor } \\
\hline \multicolumn{8}{|c|}{ Adjusted Akaike Information Criterion } \\
\hline \multirow{2}{*}{\multicolumn{8}{|c|}{ Bayesian Information Criterion }} \\
\hline \multicolumn{4}{|c|}{ Hannan-Quinn information criterion } & & & & \\
\hline
\end{tabular}

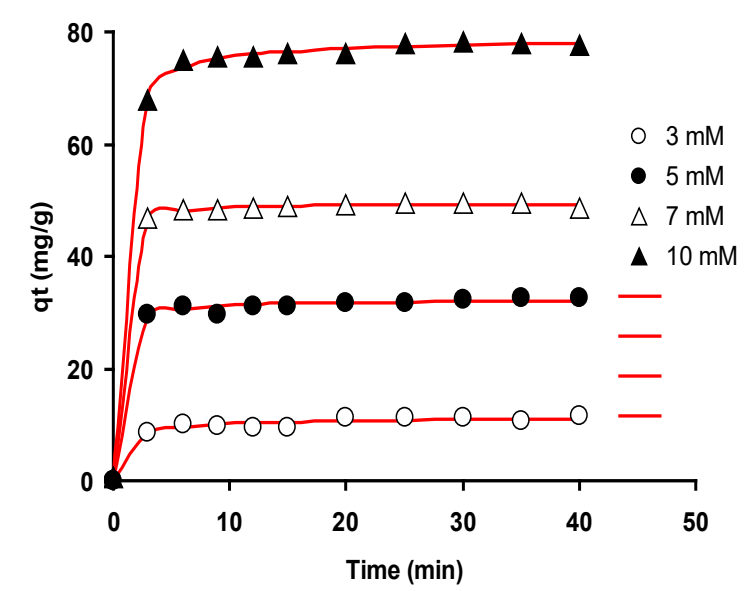

Fig. 4. Fitting of the kinetics of the sorption of Malachite Green at various concentrations onto graphene oxide as modelled using the pseudo- $2^{\text {nd }}$ order model.

Using kinetic models to examine experimental data, researchers have been able to better understand the mechanism of sorption as well as probable rate-controlling phases, such as chemical reactions and mass transport mechanisms. Incorporating the pseudo-1st order equation, the pseudo-2nd order equation, and the Elovich equation, these kinetic models yielded the best results. Before the pseudo first-order process can proceed, the adsorbate concentration must be saturated. This has the effect of maintaining the adsorbate concentration at a constant level, which in turn ensures that the adsorbate is absorbed at a steady rate. The rate is inversely proportional to particle size, distribution coefficient, and film thickness when it is controlled by film diffusion. If both reactants are present in the solution, then the rate-limiting phase of diffusion will be referred to as physisorption (physical exchange) [22-25].

The pseudo- $2^{\text {nd }}$ order has been found to be the best model in several sorption works involving malachite green such as in the biosorption of Malachite Green from aqueous solutions onto aerobic granules [26], malachite green biosorption onto beech sawdust [4] and malachite green sorption from simulated wastewater onto Carica papaya wood biosorbent [27]. The ratecontrolling step is controlled by the chemical reaction when it is driven by a pseudo second order reaction, and this is referred to as chemisorption (chemical absorption). A second-order reaction happens when this occurs at low adsorbate/adsorbent ratio and two competing second-order reactions happen at higher adsorbate/selective sorbent ratios [28]. However, additional evidence is needed to support this conclusion, such as the evaluation of activation energies obtained by re-running the experiment at various temperatures, and an examination of procedure rates in regards to adsorbent particle size, as well as their reliance on the adsorbent's particle size [29].

\section{CONCLUSION}

In conclusion, the Malachite Green onto graphene oxide was successfully modelled using three models - pseudo- ${ }^{\text {st }}$, pseudo$2^{\text {nd }}$ and Elovich, and fitted using non-linear regression. Statistical analysis based on root-mean-square error (RMSE), adjusted coefficient of determination $\left(\operatorname{adj} R^{2}\right)$, bias factor $(\mathrm{BF})$, accuracy factor (AF), corrected AICc (Akaike Information Criterion), Bayesian Information Criterion (BIC) and Hannan-Quinn information criterion (HQC) showed that the pseudo- $2^{\text {nd }}$ order model was the best model. Nonlinear regression analysis using the pseudo- $2^{\text {nd }}$ order model gave values of equilibrium sorption capacity $q_{e}$ of $6.164 \mathrm{mg} / \mathrm{g}$ (95\% confidence interval from 5.918 to 6.410$)$ and a value of the pseudo- $2^{\text {nd }}$-order rate constant, $k_{2}$ of 0.034 (95\% confidence interval from 0.024 to 0.045$)$. Further analysis is needed to provide proof for the mechanism usually tied to this kinetic. The nonlinear regression method allows for the parameter values to be represented in the $95 \%$ confidence interval range which can better allow comparison with published results.

\section{REFERENCES}

1. Srivastava S, Sinha R, Roy D. Toxicological effects of malachite green. Aquat Toxicol. 2004;66(3):319-29.

2. Guerra-Lopez D, Daniels L, Rawat M. Mycobacterium smegmatis mc2 155 fbiC and MSMEG_2392 are involved in triphenylmethane dye decolorization and coenzyme F420 biosynthesis. Microbiology. 2007;153(8):2724-32.

3. Chowdhury S, Mishra R, Saha P, Kushwaha P. Adsorption thermodynamics, kinetics and isosteric heat of adsorption of malachite green onto chemically modified rice husk. Desalination. 2011;265(1-3):159-68.

4. Witek-Krowiak A. Analysis of influence of process conditions on kinetics of malachite green biosorption onto beech sawdust. Chem Eng J. 2011 Jul 15;171(3):976-85.

5. Khattri SD, Singh MK. Colour removal from synthetic dye wastewater using a bioadsorbent. Water Air Soil Pollut. 2000;120(3-4):283-94.

6. Myung HK, Kim Y, Park H-J, Jong SL, Kwak S-N, Jung W-H, et al. Structural insight into bioremediation of triphenylmethane dyes by Citrobacter sp. triphenylmethane reductase. J Biol Chem. 2008;283(46):31981-90.

7. Lian Z, Wang J. Molecularly imprinted polymer for selective extraction of malachite green from seawater and seafood coupled with high-performance liquid chromatographic determination. Mar Pollut Bull. 2012 Dec 1;64(12):2656-62.

8. Dhananjaneyulu BV, Kumaraswamy K. Kinetic and thermodynamic studies on adsorption of malachite green from 
aqueous solution using mixed adsorbents (Rice husk and egg shell). Res J Pharm Technol. 2016;9(10):1671-6.

9. Naeem H, Ajmal M, Muntha S, Ambreen J, Siddiq M. Synthesis and characterization of graphene oxide sheets integrated with gold nanoparticles and their applications to adsorptive removal and catalytic reduction of water contaminants. RSC Adv. 2018;8(7):3599-610.

10. Motulsky HJ, Ransnas LA. Fitting curves to data using nonlinear regression: a practical and nonmathematical review. FASEB J Off Publ Fed Am Soc Exp Biol. 1987;1(5):365-74.

11. Tran HN, You S-J, Hosseini-Bandegharaei A, Chao H-P. Mistakes and inconsistencies regarding adsorption of contaminants from aqueous solutions: A critical review. Water Res. 2017 Sep $1 ; 120: 88-116$

12. Rohatgi

$$
\text { A. }
$$

WebPlotDigitizer. http://arohatgi.info/WebPlotDigitizer/app/ Accessed June 2 2014.; 2015.

13. Halmi MIE, Shukor MS, Johari WLW, Shukor MY. Mathematical modelling of the degradation kinetics of Bacillus cereus grown on phenol. J Environ Bioremediation Toxicol. 2014;2(1):1-5.

14. Khare KS, Phelan Jr FR. Quantitative comparison of atomistic simulations with experiment for a cross-linked epoxy: A specific volume-cooling rate analysis. Macromolecules. 2018;51(2):56475.

15. Lagergren S. Zur theorie der sogenannten adsorption gelöster stoffe (About the theory of so-called adsorption of soluble substances). K Sven Vetenskapsakademiens Handl. 1898;24(4):1-39.

16. Ho YS, McKay G. Pseudo-second order model for sorption processes. Process Biochem. 1999 Jul 1;34(5):451-65.

17. Zeldovich J. Über den mechanismus der katalytischen oxydation von CO an $\mathrm{MnO}_{2}$. Acta Physicochim URSS. 1934;1:364-499.

18. Burnham KP, Anderson DR. Model Selection and Multimodel Inference: A Practical Information-Theoretic Approach. Springer Science \& Business Media; 2002. 528 p.

19. Akaike H. New look at the statistical model identification. IEEE Trans Autom Control. 1974;AC-19(6):716-23.

20. Kass RE, Raftery AE. Bayes Factors. J Am Stat Assoc. 1995 Jun 1;90(430):773-95.

21. Ross T, McMeekin TA. Predictive microbiology. Int J Food Microbiol. 1994;23(3-4):241-64.

22. Yuan G, Zhao B, Chu KH. Adsorption of fluoride by porous adsorbents: Estimating pore diffusion coefficients from batch kinetic data. Environ Eng Res. 2020;25(5):645-51.

23. Li H, He J, Chen K, Shi Z, Li M, Guo P, et al. Author Response to Comment on: Dynamic Adsorption of Sulfamethoxazole from Aqueous Solution by Lignite Activated Coke. Materials. 2021 Jan;14(4):868.

24. Hu Q, Pang S, Wang D. In-depth Insights into Mathematical Characteristics, Selection Criteria and Common Mistakes of Adsorption Kinetic Models: A Critical Review. Sep Purif Rev. 2021 Jul 1;0(0):1-19.

25. González-López ME, Laureano-Anzaldo CM, Pérez-Fonseca AA, Arellano M, Robledo-Ortíz JR. A Critical Overview of Adsorption Models Linearization: Methodological and Statistical Inconsistencies. Sep Purif Rev. 2021 Aug 1;0(0):1-15.

26. Sun X-F, Wang S-G, Liu X-W, Gong W-X, Bao N, Gao B-Y, et al. Biosorption of Malachite Green from aqueous solutions onto aerobic granules: Kinetic and equilibrium studies. Bioresour Technol. 2008 Jun 1;99(9):3475-83.

27. S R, Lata S, P B. Biosorption characteristics of methylene blue and malachite green from simulated wastewater onto Carica papaya wood biosorbent. Surf Interfaces. 2018 Mar 1;10:197-215.

28. Qurie M, Khamis M, Manassra A, Ayyad I, Nir S, Scrano L, et al. Removal of $\mathrm{Cr}(\mathrm{VI})$ from aqueous environments using micelle-clay adsorption. Sci World J. 2013;Article ID 942703:7.

29. Khamizov RKh, Sveshnikova DA, Kucherova AE, Sinyaeva LA. Kinetic model of batch sorption processes: Comparing calculated and experimental data. Russ J Phys Chem A. 2018 Oct 1;92:20328 . 